\title{
Structure resonances due to space charge in periodic focusing channels
}

\author{
Chao $\mathrm{Li}^{*}$ \\ Key Laboratory of Particle Acceleration Physics and Technology, Institute of High Energy Physics, \\ Chinese Academy of Sciences, 19(B) Yuquan Road, Beijing 100049, China \\ and Institut für Kernphysik-4, Forschungszentrum Jülich, 52425 Jülich, Germany \\ R. A. Jameson \\ Institut für Angewandte Physik, Goethe Uni Frankfurt, 60323 Frankfurt-am-Main, Germany
}

(Received 8 October 2017; published 21 February 2018)

\begin{abstract}
The Vlasov-Poisson model is one of the most effective methods to study the space charge dominated beam evolution self-consistently in a periodic focusing channel. Since the approach to get the solution with this model is not trivial, previous studies are limited in degenerated conditions, either in smoothed channel (constant focusing) [I. Hofmann, Phys. Rev. E 57, 4713 (1998)] or in alternating gradient focusing channel with equal initial beam emittance condition in the degrees of freedom [I. Hofmann et al., Part. Accel. 13, 145 (1983); Chao Li et al., THOBA02, IPAC2016]. To establish a basis, we intentionally limit this article to the study of the pure transverse periodic focusing lattice with arbitrary initial beam condition, and the same lattice structure in both degrees of freedom, but with possibility of different focusing strengths. This will show the extension of the existing work. The full Hamiltonian is invoked for a pure transverse focusing lattice in various initial beam conditions, revealing different mode structure and additional modes beyond those of the degenerated cases. Application of the extended method to realistic lattices (including longitudinal accelerating elements) and further details will then reveal many new insights, and will be presented in later work.

DOI: 10.1103/PhysRevAccelBeams.21.024204
\end{abstract}

\section{INTRODUCTION}

A fundamental understanding of the equilibrium and stability properties of high intensity beam in linear and circular accelerators is crucial for the development of advanced particle accelerator applications [1-3]. Thus, great attention has been paid in the past to the collective beam instability caused by space charge in high intensity machines. From the analytical point of view, the stability study of the intense beam requires a self-consistent process in which the Vlasov and Poission equations have to be satisfied simultaneously. Researches following the selfconsistent Vlasov-Poisson description are known as collective beam dynamics [4-6]. The solution for such coupled Vlasov-Poisson system is not trivial and the researches on the subject started from simplified approaches and models, typical examples are systems with low degrees of freedom and smooth approximation leading to a constant focusing channel assumption. As to the smooth approximation-the constant focusing channel,

\footnotetext{
*lichao@ihep.ac.cn

Published by the American Physical Society under the terms of the Creative Commons Attribution 4.0 International license. Further distribution of this work must maintain attribution to the author(s) and the published article's title, journal citation, and DOI.
}

the oscillating mode of a uniform 2D beam with only one degree of freedom was first studied by Sacherer and Smith $[7,8]$, in which the dispersion relationship was derived to show the unstable parameter region indicated by the existence of an imaginary part of the eigenfrequency. In the 1970s, R. L. Gluckstern extended Sacherer's work to the beam system with two degrees of freedom $[9,10]$, assuming 4D Kapchinskij-Vladimirskij (KV) input beam distribution [11], but limited to the specific condition that the external focusing forces are constant and the emittances in two degrees of freedom are equal. Hofmann extended Gluckstern's work to various initial beam emittance ratios in two degrees of freedom and gave the general dispersion relationship formulation when different orders of perturbation due to space charge of the initial distribution are injected into a constant focusing channel [6].

However, smooth approximation removes all details inside the transverse focusing period and nearly all actual ring and linear accelerator lattices contain details inside the transverse focusing period that we want to understand, such as, the separated functionality of the actual transport, inclusion of driving functions other than just transverse focusing elements, etc.

In a nonsmoothed periodic alternative gradient focusing channel, the collective modes based on the Vlasov-Poisson approach and 4D KV initial beam assumption were first studied by Laslett and Smith [12] at Lawrence Berkeley 
Laboratory. The first general report is given by Hofmann et al. in 1983 [4] and revisited by Chao Li et al. in 2016 [5]. However, these results are for a limited case with equal external focusing and emittances in two degrees of freedom.

To establish a basis, we intentionally limit this article to the study of the pure transverse periodic focusing lattice. This will show the extension of the existing work. The full Hamiltonian is invoked for a pure transverse focusing lattice, revealing different mode structure and additional modes beyond those obtained from the smooth approximation [6] and those from limited beam initial conditions $[4,5,12]$. Application of the extended method to realistic lattices (including longitudinal accelerating elements) and further details will then reveal many new insights, and will be presented in later work.

In this paper, following the former studies [5], the Vlasov-Poisson model is extended to pure transverse periodic focusing channels with no smooth approximation and arbitrary emittance ratio between two degrees of freedom. The eigenmodes up to the 4th order are analytically constructed and eigenvalues are numerically obtained for collective stability analysis. Since the KV beam initial distribution supplies only linear space charge inside the beam and lacks detuning and damping mechanisms except only from numerical noise, conclusions obtained from KV beam analysis have to be carefully checked to extend to other beam distributions. In Sec. II, we carefully define the terminologies of different kinds of resonances we identify in this paper, and specify the structure resonance mechanisms we will mainly discuss. In Sec. III, the basic idea to construct collective modes and the mathematical approach to get the related eigenvalues are given. The parameter region where structure resonance takes place is termed as collective unstable stop band. In Sec. IV, we show that results obtained degenerate to results discussed in Ref. [6] if the smooth approximation is adopted. Discussion is given in Sec. V.

\section{NOMENCLATURE OF RESONANCES IN ION ACCELERATORS}

In periodical dynamics system with period length $S$, $Z(s+S)=M(S) Z(s)$, the term "phase advance" is defined as the phase that the oscillator sweeps in phase space $\left(Z(s), Z^{\prime}(s)\right) ; M(S)$ is usually defined as the transfer matrix requiring $M(s)=M(s+S)$. In a general sense, the eigenvalues of transfer matrix $\operatorname{Abs}[M(S)]$ indicates the stability of the system and the eigenphase $\operatorname{Arg}[M(S)]$ gives the related phase advance. In ion accelerators, we focus on three motions: single particle motion $x(s)$, beam envelope oscillation $R(s)$, and collective motion $I_{j ; k, l}(s)$ (defined in Sec. III), which have to be carefully evaluated simultaneously. As a consequence, three types of "phase advance" can be defined in the isolated phase spaces $\left(x(s), x^{\prime}(s)\right)$, $\left[R(s), R^{\prime}(s)\right]$, and $\left[I_{j ; k, l}(s), I_{j ; k, l}^{\prime}(s)\right]$ respectively.
Single particle phase advance $\sigma_{s}$ is used for single particle dynamics. The nonlinear effects from external elements and internal space charge cause different particles to have different particle phase advance $\sigma_{s}$, leading to a beam phase advance spread. In rings, this phase advance spread in equivalent to tune spread.

Envelope phase advance $\Phi_{e}$ is used to depict the envelope $R(s)$ oscillation characteristics. In a periodic channel with matched beam condition, the envelope oscillates once in one focusing period, which makes one whole elliptical trajectory in $\left(R, R^{\prime}\right)$ phase space. Thus this envelope phase advance $\Phi_{e}=360^{\circ} . \Phi_{e}$ naturally represents the periodicity of the lattice. The words "envelope oscillation period" and "lattice period" are considered equivalent.

Collective mode phase advance $\Phi_{j ; k, l}$ is used to depict the collective modes $I_{j ; k, l}$. In this manuscript, one of the key issues is to model the collective motions and to show how to get these collective mode phase advances $-\Phi_{j ; k, l}$.

Phase advance with and without beam current $\sigma_{0}, \sigma$ are the standard notation; $\sigma_{0}$ and $\sigma$ are used to evaluate the average focusing strength in one focusing period without and with space charge. They play the same role as working point and detuned working point in rings. In periodic linacs, they are obtained from the integration of the betatron function numerically.

In general, the terminology resonance is expressed as ratios between normalized frequencies in the form $\omega_{1} / \omega_{2}=$ $n / m$, where $m$ and $n$ are arbitrary integers. If appropriate driving forces were applied on such systems, driven resonances take place between the two motions. In a simplified periodic lattice, normalized by the period length $S$, three frequencies can be defined: $\omega_{s}=\sigma_{s} / 360^{\circ}, \omega_{e}=$ $\Phi_{e} / 360^{\circ}=1$, and $\omega_{j ; k, l}=\Phi_{j ; k, l} / 360^{\circ}$ due to motions of single particle motion, envelope and collective modes. Since we are mainly concentrating on the collective beam behavior, resonances related to single particle behavior $\sigma_{s}$ will not be discussed in the paper but in a separate research on beam halo formation. Thus in the following, we will mainly discuss the resonances between any two collective modes $\Phi_{j ; k, l}$ or between any $\Phi_{j ; k, l}$ and $\Phi_{e}$.

Following the former study of envelope dynamics $[13,14]$, the collective unstable stop band is derived from parametric resonance with the condition $\Phi_{j ; k, l} / \Phi_{e}=n / 2$ or confluent resonance when two eigenphases satisfy $\Phi_{j ; k, l}^{1}+$ $\Phi_{j ; k, l}^{2}=n \Phi_{e}$. We adopt the terminology structure resonance to include both parametric resonances and confluent resonances. Physically, we emphasize again that the parametric resonance refers in particular to resonance between collective mode $\Phi_{j ; k, l}$ and lattice period $\Phi_{e}$; whereas the confluent resonance refers in particular to resonance between different collective modes $\Phi_{j ; k, l}$. Generally, parametric resonance is indicated by eigenphase $\Phi_{j ; k, l}$ locking to $n * 180^{\circ}$ lines and confluent resonance is indicated by the merging of two eigenphases. The collective motions can be 
grouped into positive energy oscillation modes and negative energy oscillation modes. It is believed that when the (positive-negative) energy oscillation modes merge, it is a peculiarity of the KV beam and these unstable modes are usually ignored.

\section{COLLECTIVE MODES ANALYSIS WITH VLASOV-POISSON MODEL}

In this section, we summarize the basic methods and approaches to get the collective modes based on the coupled Vlasov-Poisson model. Following the former researches, the $4 \mathrm{D} \mathrm{KV}$ (the only known beam profile that is self-consistent and can be treated analytically in linear channel) initial beam evolution in a periodic linear focusing channel-coasting beam-is used to model the beam behavior. The classical linearized perturbation theory [15] is used to obtain the collective motions-eigenmodes. Defining the KV beam function in phase space as $f_{0}\left(H_{0}\right)$, and the unperturbed particle Hamiltonian is noted as $H_{0}$, equilibrium condition requires

$$
\begin{aligned}
f_{0}\left(x, p_{x}, y, p_{y}\right)= & f\left(H_{0}\right), \\
H_{0}= & \frac{1}{2}\left[k_{x}(s) x^{2}+p_{x}^{2}\right]+\frac{1}{2}\left[k_{y}(s) y^{2}+p_{y}^{2}\right] \\
& +V_{s c}(x, y)
\end{aligned}
$$

where $k_{x}(s)$ and $k_{y}(s)$ represent the external focusing strength supplied by the focusing elements; $p_{x}$ and $p_{y}$ are generalized momentum in $x$ and $y$ direction; $V_{s c}(x, y)$ is the space charge potential without any perturbation. The distribution function $f_{0}$ has to meet the Vlasov equation and space charge potential has to meet the Poisson equation that

$\frac{\partial f_{0}}{\partial s}+\left[f_{0}, H_{0}\right]=0, \quad \nabla^{2} V_{s c}(x, y)=\frac{1}{\epsilon_{0}} \iint f_{0} d x d y$,

where [,] is the Poisson bracket operator. The existence of an arbitrary perturbation $f_{1}$ will lead to the perturbed space charge potential $V_{s c}^{1}(x, y ; s)$. Thus, the first order linearized Vlasov and Poisson equation can be obtained

$$
\begin{aligned}
\frac{\partial f_{1}}{\partial s}+\left[f_{1}, H_{0}\right]+\left[f_{0}, V_{s c}^{1}\right] & =0, \\
\nabla^{2} V_{s c}^{1}(x, y) & =\frac{1}{\epsilon_{0}} \iint f_{1} d x d y .
\end{aligned}
$$

which indicates the evolution of perturbation $f_{1}$ will be affected both by the space charge potential $V_{s c}$ generated by $f_{0}$ and perturbed space charge potential $V_{s c}^{1}$ generated by $f_{1}$. Meanwhile, $\left(f_{0} V_{s c}\right)$ and $\left(f_{1} V_{s c}^{1}\right)$ both have to meet the Poisson equation and boundary conditions to ensure the self-consistence. Thus, with appropriate coupled equations constructed, the eigenmodes analysis of the perturbed system can be used to check if $f_{1}$ would be self-motivated and self-magnified by the space charge potential $V_{s c}^{1}$ generated by $f_{1}$ itself-if so, this would constitute an instability.

Generally, it is assumed that the perturbed space charge potential function is polynomial form in the interior of the beam. This actually assumes that the beam surface is distorted in real space by the space charge potential to a regular pattern. It will be shown that with different perturbation potentials $V_{s c}^{1}(x, y ; s)$, various collective motions $I_{j ; k, l}(s)$-definition as shown in Eq. (9)—can be constructed, which physically represent the integrals of the surface electric field discontinuity from period to period. To ensure self-consistence, the constructed $I_{j ; k, l}(s)$ have to meet the Poisson equation and boundary conditions, and finally the transfer of the collective motions $I(s)=\left[I_{j ; k, l}(s), I_{j ; k, l}(s)^{\prime}\right]$ in one period is in the form $I^{\prime}(s)=M(s) I(s)$, which is exactly the Mathieu equation. The stability of the system $I(s)=M(s) I(s)$ is decided with the eigenvalues $\lambda$ of the Jacobi matrix $M(S)$ in one period (Floquet's theory). In a general case, with $N$ constructed integrals $I_{j ; k, l}(s)$, the obtained $2 N * 2 N$ Jacobi matrix $M(s)$ can be grouped into $\mathrm{N}$ pairs of eigenvalues $\lambda_{i}, \lambda_{i}^{*}$ and eigenphases $\Phi_{i}, \Phi_{i}^{*}$. Each pair of eigenvalues are reciprocal pairs and complex conjugates that $\left|\lambda_{i}\right|=1 /\left|\lambda_{i}^{*}\right|, \quad \Phi_{i}+$ $\Phi_{i}^{*}=0^{\circ}$; It means that the total $2 \mathrm{~N}$ dimension phase space composed of $\left[I_{j ; k, l}(s), I_{j ; k, l}(s)^{\prime}\right]$ is derived from a Hamiltonian system and has to meet the Liouville theorem. If the phase space in one direction is stretched, the other is always compressed. Fig. 1 shows the possible eigenvalues for map $M(S)$. Whenever the structure resonance takes place, the eigenvalues will leave the unit circle with the condition $\lambda \neq 1$-collective instability. Since $\Phi_{j ; k, l}$ and
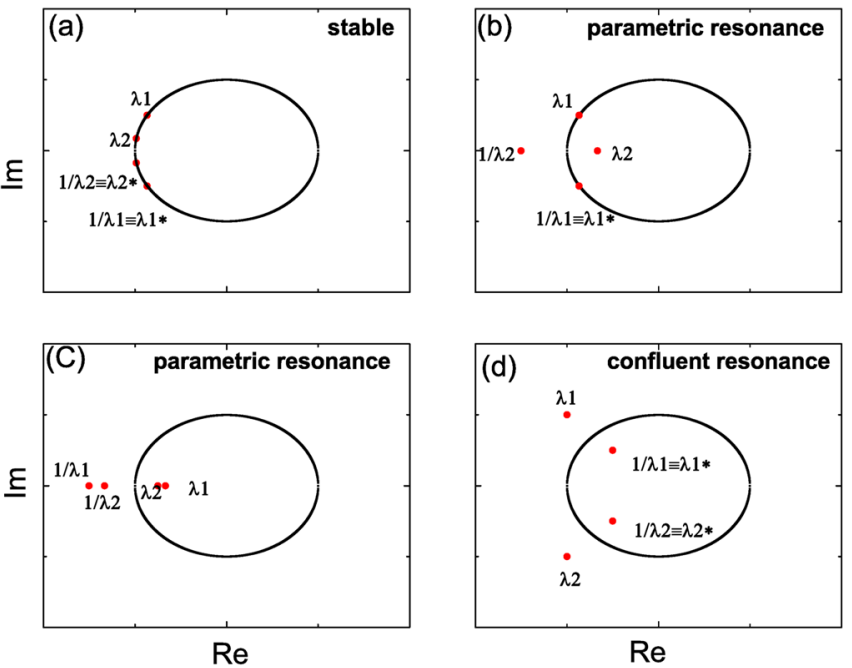

FIG. 1. Locations of possible eigenvalues for map $M(S)$, where $S$ is period length. 
$\Phi_{j ; k, l}^{*}$ describe the same physics, in the following, only $\Phi_{j ; k, l}>0^{\circ}$ is kept for discussion.

\section{A. Collective modes in time dependent periodic channel}

Hofmann et al. showed the basic mathematical approaches to construct the dynamics caused by the perturbation $f_{1}$ in periodic focusing channels, limited to equal emittance and equal external focusing in two degrees of freedom in Ref. [4]. Here, with similar method, we extend the mathematical approaches to cover problems with various beam conditions by citing a new parameter noted as emittance ratio $\Gamma=\epsilon_{y} / \epsilon_{x}$ in two degrees of freedom and retain the nonsmoothed condition-full time (position) dependent. In the following, we introduce the basic model and approaches briefly.

Denoting $s$ as the longitudinal variable (physically, $s$ plays the role as time here) along the channel, with Courant-Snyder transformation, the single particle Hamiltonian Eq. (1) can be normalized in the form

$H=\frac{1}{2 \beta_{x}}\left(p_{x}^{2}+x^{2}\right)+\frac{\Gamma}{2 \beta_{y}}\left(p_{y}^{2}+y^{2}\right)+\frac{V_{s c}^{1}(x, y ; s)}{\epsilon_{x}}$,

where $V_{s c}^{1}(x, y ; s)$ denotes the generalized electrostatic space charge potential perturbation function on the ideal self-consistent KV beam. The KV distribution function has the form

$f_{0}=\frac{N}{\pi^{2} \Gamma} \delta\left(x^{2}+p_{x}^{2}+\frac{y^{2}+p_{y}^{2}}{\Gamma}-1\right), \quad \Gamma=\epsilon_{y} / \epsilon_{x}$,

where $\delta$ denotes the Dirac delta function. Note $f_{1}=\delta f_{0}$ is the perturbation of the density distribution function. For clarity and simplicity, we inherit the symbols used in Ref. [4], skip the tedious mathematics and just show the basic ideas and indispensable equations here.

Physically, there are only two sets of constraint conditions that the system is subject to. The first one is the Poisson equation Eq. (3), which shows that the perturbation $f_{1}$ and the related perturbed space charge potential $V_{s c}^{1}(x, y ; s)$ have to be treated consistently. The second set of constraint equations requires the continuity of the space charge electric field at the beam boundary. From one side, the electric field jump is expressed as

$$
\begin{aligned}
\Delta \frac{\partial V_{s c}^{1}}{\partial \xi}= & \frac{Q}{\epsilon_{x}} \int_{0}^{s}\left(\frac{\partial}{\partial \psi_{x}^{\prime}}+\frac{1}{\Gamma} \frac{\partial}{\partial \psi_{y}^{\prime}}\right) V_{s c}^{1} \\
& \times\left[\cos \zeta \cos \left(\psi_{x}^{\prime}-\psi_{x}\right),\right. \\
& \left.\sqrt{\Gamma} \sin \zeta \cos \left(\psi_{y}^{\prime}-\psi_{y}\right) ; s^{\prime}\right] d s^{\prime} .
\end{aligned}
$$

in elliptical coordinate system. From the other side, assuming the space charge potential $V_{s c}^{1}(x, y ; s)$ inside the beam up to the $n$th order in form of
$\left(V_{s c}^{1}\right)_{n}=\sum_{m=0}^{n} A_{m}(s) x^{n-m} y^{m}+\sum_{m=0}^{n-2} A_{m}^{(1)}(s) x^{n-m} y^{m}+\cdots ;$

the solution for perturbed space charge potential $V_{s c}^{1}(x, y ; s)$ outside of the beam is a superposition of angular harmonics which vanish at infinity as

$$
e^{-l\left(\xi-\xi_{0}\right)} \cos l \zeta, \quad e^{-l\left(\xi-\xi_{0}\right)} \sin l \zeta .
$$

Combining the Eqs. (6), (7), (8) together, the second set of constraint equations can be derived. Finally, the constructed integrals $I_{j ; k, l}(s)$, composing of the dynamic system and representing the motion of the collective modes, are expressed with the general form

$I_{j ; k, l}(s)=\int_{0}^{s} A_{j}\left(s^{\prime}\right) \sin \left[k\left(\psi_{x}^{\prime}-\psi_{x}\right)-l\left(\psi_{y}^{\prime}-\psi_{y}\right)\right] d s^{\prime} ;$

The dynamics equation that the $I_{j ; k, l}$ have to obey is

$\frac{1}{C_{k, l}(s)} \frac{d}{d s}\left[\frac{1}{C_{k, l}(s)} \frac{d I_{j ; k, l}(s)}{d s}\right]+I_{j ; k, l}(s)=-\frac{1}{C_{k, l}(s)} A_{j}(s)$.

where $C_{k, l}(s)=\left[\frac{k}{\beta_{x}(s)}+\frac{l}{\beta_{y}(s)}\right]$.

In the following, $V_{s c}^{1}$ will be replaced by $V$ for simplicity. It is noteworthy that for a given order $n$ in Eq. (7), the even mode and odd mode, which directly represents the tilt of the beam elliptical distribution in real space, conveniently may be treated separately, on the basis of whether the index $m$ is restricted to even or to odd integer values.

\section{B. General treatment of collective modes}

\section{The 1st order modes}

For the 1st order mode, the perturbed space charge potential inside near the beam boundary is assumed as $V_{1}=A_{1}(s) x$ (or $\left.V_{1}=A_{1}(s) y\right)$. The influence of the 1st order mode will be recognized as a simple oscillation of the beam as a whole [16]. It does not lead to any collective instability.

\section{The 2nd order modes}

For the 2nd order mode, the related perturbed space charge potential inside near the beam boundary is in the form $V_{2 e}=A_{0}(s) x^{2}+A_{2}(s) y^{2}$ and $V_{2 o}=A_{1}(s) x y$ respectively, where subscript $e$ and $o$ refer to even and odd modes. Table I gives the explicit forms of the eigenphases with and without beam current, structure resonance driving terms, and the related forms of the perturbed space charge potential. Figure 2 shows the eigenvalues and eigenphases 
TABLE I. 2nd order eigenmodes, eigenphases, and their driving terms.

\begin{tabular}{lcccc}
\hline \hline Periodic integral & Eigenphases & Driving term & Eigenphases (zero current) & Perturbed potential \\
\hline$I_{0 ; 2,0}$ & $\Phi_{0 ; 2,0}$ & $\cos 2\left(\psi_{x}^{\prime}-\psi_{x}\right)$ & $2 \sigma_{x 0}$ & $A_{0}(s) x^{2}$ \\
$I_{2 ; 0,2}$ & $\cos 2\left(\psi_{y}^{\prime}-\psi_{y}\right)$ & $2 \sigma_{y 0}$ & $A_{2}(s) y^{2}$ \\
$I_{1 ; 1,1}$ & $\Phi_{1 ; 1,1}$ & $\cos \left[\left(\psi_{x}^{\prime}-\psi_{x}\right)+\left(\psi_{y}^{\prime}-\psi_{y}\right)\right]$ & $\sigma_{x 0}+\sigma_{y 0}$ & $A_{1}(s) x y$ \\
$I_{1 ; 1,-1}$ & $\Phi_{1 ; 1,-1}$ & $\cos \left[\left(\psi_{x}^{\prime}-\psi_{x}\right)-\left(\psi_{y}^{\prime}-\psi_{y}\right)\right]$ & $\sigma_{x 0}-\sigma_{y 0}$ & $A_{1}(s) x y$ \\
\hline \hline
\end{tabular}

evolution when the space charge effect is increased (right to left) for the 2nd order mode. Compared with the case $\sigma_{x}=\sigma_{y}, \Gamma=1$ [14], where only confluent resonance can be excited, here both the confluent resonance and parametric resonance can be excited. Clearly, since the FODO channel used here is limited to $\sigma_{x 0}=\sigma_{y 0}=\sigma_{0}$, once $\sigma_{0}<90^{\circ}$, the 2 nd order even mode instability can be avoided. For the 2 nd odd mode, with the initial condition we chose, the $\Phi_{1 ; 1,1}$ just slightly passes through the $180^{\circ}$ line and will not lead to any instability.

It is worthwhile to point out that the well-known envelope instability stop band obtained from the perturbed envelope dynamics actually gives exactly the same result as the 2 nd order even mode. It is not surprising, because the dynamics of the perturbed envelope equations exactly describes the same physics as the 2 nd order even mode, although they are derived with different basic assumptions and approaches. The $\Phi_{0 ; 2,0}$ that touches the $180^{\circ}$ line faster is also named the quadrupole mode; the slower one $\Phi_{2 ; 0,2}$ is named the breathing mode. It has been proved that this instability in particularly refer to a bifurcation process [17] in a degenerate 1D envelope dynamics. Here, we suppose that bifurcation process can be extended to instabilities due to higher order modes.

\section{The 3rd order and 4th order modes}

As suggested by Eq. (7), the perturbed space charge potential inside the beam for the 3rd and 4th order can be obtained explicitly. Similar process is adopted to construct the periodic discontinuity of the electric field $I_{j ; k, l}(s)$. It is important to point out that the lower order perturbation $V_{n-2}$ has to be contained in the $n$th order perturbation $V_{n}$ to

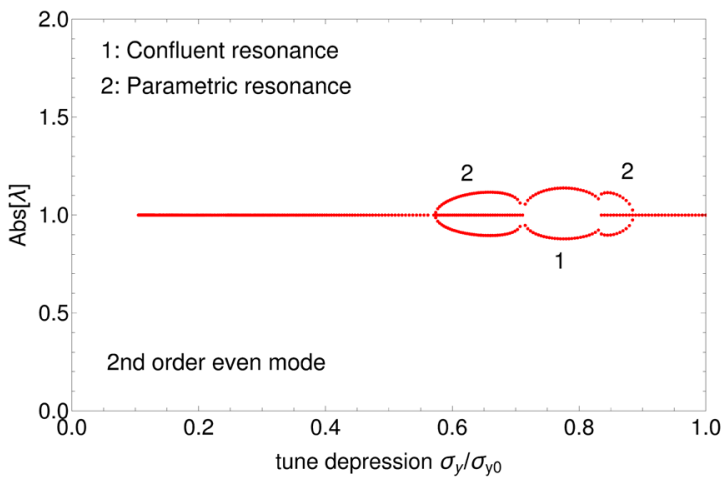

(a)

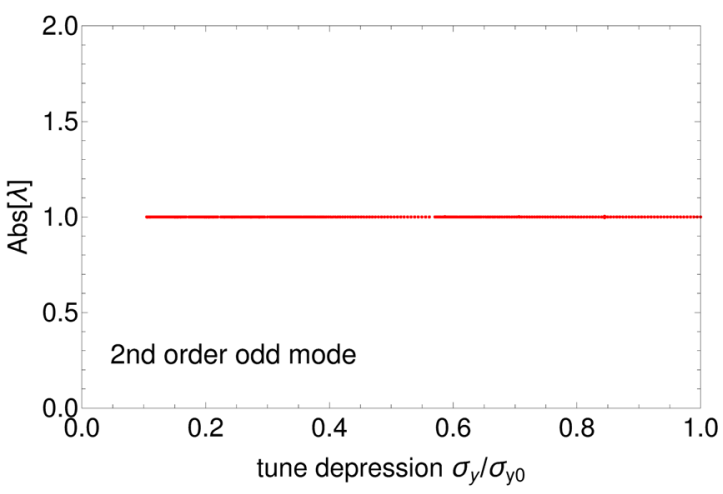

(c)

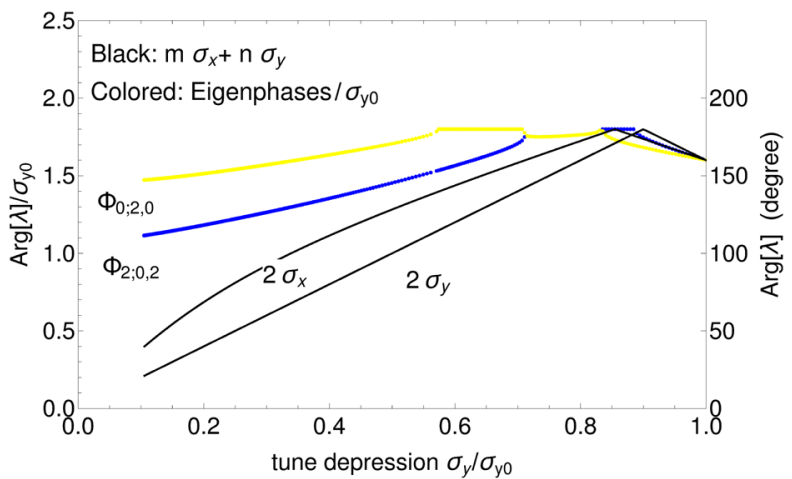

(b)

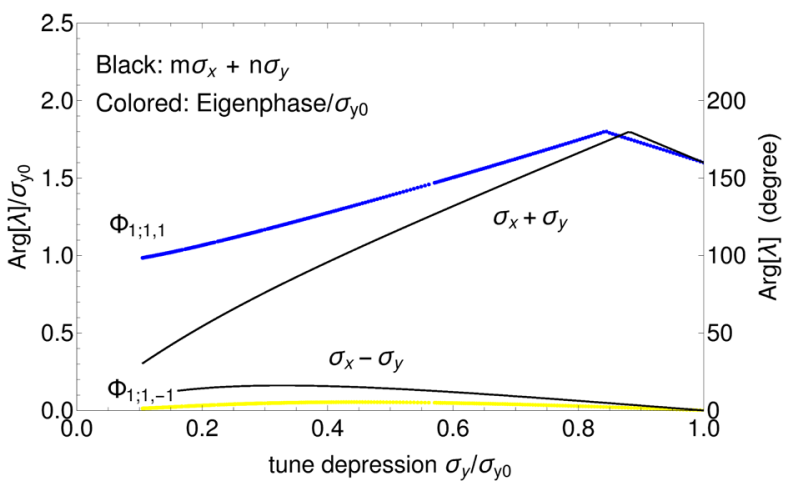

(d)

FIG. 2. Eigenvalues $A b s|\lambda|$ and eigenphases $A r g|\lambda|$ of the 2nd even mode, (a) and (b), and odd mode, (c) and (d) $v s$ tune depression $\sigma_{y} / \sigma_{y 0}$, for a FODO channel with the parameter $\sigma_{x 0}=\sigma_{y 0}=100^{\circ}$, emittance ratio $\Gamma=1 / 2$, filling factor $\eta=0.5$. 
TABLE II. 3rd order even eigenmodes, eigenphases, and their driving terms.

\begin{tabular}{lcccc}
\hline \hline Periodic integral & Eigenphases & Driving term & Eigenphases (zero current) & Perturbed potential \\
\hline$I_{0 ; 1,0}$ & $\Phi_{0 ; 1,0}$ & $\cos \left(\psi_{x}^{\prime}-\psi_{x}\right)$ & $\sigma_{x 0}$ & $A_{0}(s) x^{3}$ \\
$I_{0 ; 3,0}$ & $\Phi_{0 ; 3,0}$ & $\cos 3\left(\psi_{x}^{\prime}-\psi_{x}\right)$ & $3 \sigma_{x 0}$ & $A_{0}(s) x^{3}$ \\
$I_{2 ; 1,0}$ & $\cos \left(\psi_{x}^{\prime}-\psi_{x}\right)$ & $\sigma_{x 0}$ & $A_{2}(s) x y^{2}$ \\
$I_{2 ; 1,2}$ & $\Phi_{2 ; 1,0}$ & $\cos \left[\left(\psi_{x}^{\prime}-\psi_{x}\right)+2\left(\psi_{y}^{\prime}-\psi_{y}\right)\right]$ & $\sigma_{x 0}+2 \sigma_{y 0}$ & $A_{2}(s) x y^{2}$ \\
$I_{2 ; 1,-2}$ & $\Phi_{2 ; 1,2}$ & $\cos \left[\left(\psi_{x}^{\prime}-\psi_{x}\right)-2\left(\psi_{y}^{\prime}-\psi_{y}\right)\right]$ & $\sigma_{x 0}-2 \sigma_{y 0}$ & $A_{2}(s) x y^{2}$ \\
$I_{0 ; 1,0}^{(1)}$ & $\Phi_{2 ; 1,-2}$ & $\cos \left(\psi_{x}^{\prime}-\psi_{x}\right)$ & $\sigma_{x 0}$ & $A_{0}^{(1)}(s) x$ \\
\hline \hline
\end{tabular}

meet the boundary conditions. This indicates that the lower order stop bands are components of the higher order stop bands. To be less confusing, the order of collective instability discussed in the following refers in particular to the highest term in $V_{n}$. The reason why the effect $V_{n-2}$ could be separated will be explained latter.

Table II shows the periodic integrals $I_{j ; k, l}$, the corresponding eigenphases $\Phi_{j ; k, l}$, the forms of driving forces, and the forms of perturbed space charge potential of the $3 \mathrm{rd}$ order even mode, $V_{3 e}=A_{0}(s) x^{3}+A_{2}(s) x y^{2}+A_{0}^{(1)} x$. Six periodic perturbation integrals are constructed, which requires evaluation of the eigenvalues of a $12 \times 12$ Jacobi matrix $M(S)$. However, $M(S)$ actually is made up of a $2 \times 2$ sub-matrix, which is related to the term $I_{0 ; 1,0}^{(1)}$, and a $10 \times 10$ sub-matrix composed of other integrals. The term $I_{0 ; 1,0}^{(1)}$, particular to the potential perturbation $A_{0}^{(1)}(s) x$, does not lead to any instability. Thus, only the other 5 integrals $I_{j ; k, l}$ contribute to it. For the $3 r d$ order odd mode due to $V_{3 o}$, the unstable stop band given is the same as the even mode, because of the symmetry (interchanging $x$ and $y$ ). Figure 3 shows the 3rd order unstable stop band in FODO channel. The parametric resonances and confluent resonances are clearly identified. The stop band due to $\Phi_{0 ; 1,0}=\Phi_{2 ; 1,-2}$, which covers the whole parameter space, is a (positivenegative) energy oscillation merging mode (see Fig. 5) [18]. According to Ref. [19] the instability of such confluent (positive-negative) energy modes is a peculiarity of the $\mathrm{KV}$ distribution as a delta function distribution and such modes disappear for beams with monotonically decreasing distribution functions. Clearly, in the current case $\sigma_{0}=\sigma_{x 0}=\sigma_{y 0}$, the main third order collective unstable modes caused by parametric resonance can be avoided by choosing $\sigma_{0}<60^{\circ}$. However, if the channel has unequal external focusing $\sigma_{x 0} \neq \sigma_{y 0}$, then the parametric resonance stop band will shift and a new condition is required to avoid it.

Similarly, Table III shows the periodic integrals $I_{j ; k, l}(s)$, the corresponding eigenphases $\Phi_{j ; k, l}$, the forms of driving forces, and the forms of perturbed space charge potentials for the 4th order even mode $V_{4 e}=A_{0}(s) x^{4}+A_{2}(s) x^{2} y^{2}+$ $A_{4}(s) y^{4}+A_{0}^{(1)}(s) x^{2}+A_{2}^{(1)}(s) y^{2}$. The constructed $10 I_{j ; k, l}(s)$ result in $20 \times 20$ matrix $M(S)$, which is composed of a $4 \times 4$ sub-matrix and $16 \times 16$ sub-matrix. The $4 \times 4$ submatrix is constructed with the term $\left(I_{0 ; 2,0}^{(1)}\right)$ and $\left.I_{2 ; 0,2}^{(1)}\right)$, which comes from the perturbed potential $A_{0}^{(1)}(s) x^{2}$ and $A_{2}^{(1)}(s) y^{2}$. Not surprisingly, it gives the same unstable components as the second order even mode. In another words, the 2 nd order even mode of instability is naturedly included in the 4 th order even mode because of the requirement of beam boundary conditions. Thus, it is a fake proposition to identify which collective mode ( 2 nd even mode or 4 th even mode) takes a dominate position in the 2 nd order even mode stop band [20,21] [22]. Excluding the 2nd order

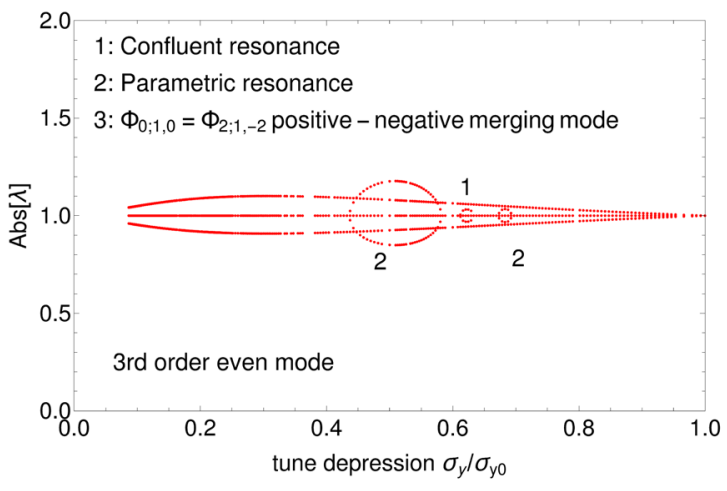

(a)

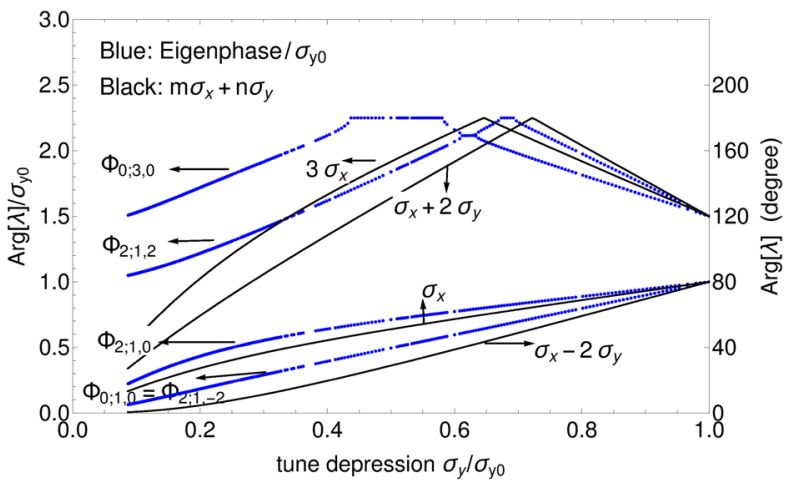

(b)

FIG. 3. Eigenvalues (a) and eigenphases (b) of the 3rd even mode $v s$ tune depression $\sigma_{y} / \sigma_{y 0}$ for FODO channel (filling factor $\eta=0.5$, emittance ratio $\Gamma=1 / 2$ ) with the parameter $\sigma_{x 0}=\sigma_{y 0}=80^{\circ}$. 
TABLE III. 4th order even eigenmodes, eigenphases, and their driving terms.

\begin{tabular}{lcccc}
\hline \hline Periodic integral & Eigenphases & Driving term & Eigenphases (zero current) & Perturbed potential \\
\hline$I_{0 ; 2,0}$ & $\Phi_{0 ; 2,0}$ & $\cos 2\left(\psi_{x}^{\prime}-\psi_{x}\right)$ & $2 \sigma_{x 0}$ & $A_{0} x^{4}$ \\
$I_{0 ; 4,0}$ & $\Phi_{0 ; 4,0}$ & $\cos 4\left(\psi_{x}^{\prime}-\psi_{x}\right)$ & $4 \sigma_{x 0}$ & $A_{0} x^{4}$ \\
$I_{2 ; 2,0}$ & $\Phi_{2 ; 2,0}$ & $\cos 2\left(\psi_{x}^{\prime}-\psi_{x}\right)$ & $2 \sigma_{x 0}$ & $A_{2} x^{2} y^{2}$ \\
$I_{2 ; 2,2}$ & $\Phi_{2 ; 2,2}$ & $\cos \left[2\left(\psi_{x}^{\prime}-\psi_{x}\right)+2\left(\psi_{y}^{\prime}-\psi_{y}\right)\right]$ & $2 \sigma_{x 0}+2 \sigma_{y 0}$ & $A_{2} x^{2} y^{2}$ \\
$I_{2 ; 2,-2}$ & $\Phi_{2 ; 2,-2}$ & $\cos \left[2\left(\psi_{x}^{\prime}-\psi_{x}\right)-2\left(\psi_{y}^{\prime}-\psi_{y}\right)\right.$ & $2 \sigma_{x 0}-2 \sigma_{y 0}$ & $A_{2} x^{2} y^{2}$ \\
$I_{2 ; 0,2}$ & $\Phi_{2 ; 0,2}$ & $\cos 2\left(\psi_{y}^{\prime}-\psi_{y}\right)$ & $2 \sigma_{y 0}$ & $A_{2} x^{2} y^{2}$ \\
$I_{4 ; 0,2}$ & $\Phi_{4 ; 0,2}$ & $\cos 2\left(\psi_{y}^{\prime}-\psi_{y}\right)$ & $2 \sigma_{y 0}$ & $A_{4} y^{4}$ \\
$I_{4 ; 0,4}$ & $\Phi_{4 ; 0,4}$ & $\cos 4\left(\psi_{y}^{\prime}-\psi_{y}\right)$ & $4 \sigma_{y 0}$ & $A_{4} y^{4}$ \\
$I_{0 ; 2,0}^{(1)}$ & $\Phi_{0 ; 2,0}^{(1)}$ & $\cos 2\left(\psi_{x}^{\prime}-\psi_{x}\right)$ & $2 \sigma_{x 0}$ & $A_{0}^{(1)} x^{2}$ \\
$I_{2 ; 0,2}^{(1)}$ & $\Phi_{2 ; 0,2}^{(1)}$ & $\cos 2\left(\psi_{y}^{\prime}-\psi_{y}\right)$ & $2 \sigma_{y 0}$ & $A_{2}^{(1)} y^{2}$ \\
\hline \hline
\end{tabular}

terms, the eigenvalues and eigenphases, obtained from the $16 \times 16$ submatrix $M(S)$, are shown in the two top subfigures in Fig. 4 with $\sigma_{x 0}=\sigma_{y 0}=80^{\circ}$ in FODO channel. The stop band caused by confluent resonance $\Phi_{4 ; 0,2}=\Phi_{2 ; 2,0}$ covering the whole parameter range, is the (positive-negative) energy oscillation merging mode. Clearly, the parametric resonance condition $\Phi_{j ; k, l}=360^{\circ}$ and $\Phi_{j ; k, l}=180^{\circ}$ both have the opportunity to be excited if $\sigma_{0}>90^{\circ}$.

Table IV shows the periodic integrals $I_{j ; k, l}$, the corresponding eigenphases $\Phi_{j ; k, l}$, the form of driving forces, and the perturbed space charge potentials for the 4th order odd mode, $V_{4 o}=A_{1}(s) x^{3} y+A_{3}(s) x y^{3}+A_{1}^{(1)}(s) x y$. The eigenvalues and eigenphases of the 4 th order odd modes are shown in the two bottom subfigures in Fig. 4 with $\sigma_{0}=\sigma_{y 0}=\sigma_{x 0}=80^{\circ}$ in FODO channel. Similar to the 4th order even mode, the 4 th order odd stop band covering the whole parametric space caused by $\Phi_{3 ; 1,1}=\Phi_{1 ; 1,1}$ is considered as a merging of (positive-negative) oscillation modes merging. The main parametric resonance stop band can be avoided with the condition $\sigma_{0}<45^{\circ}$. Again, if with

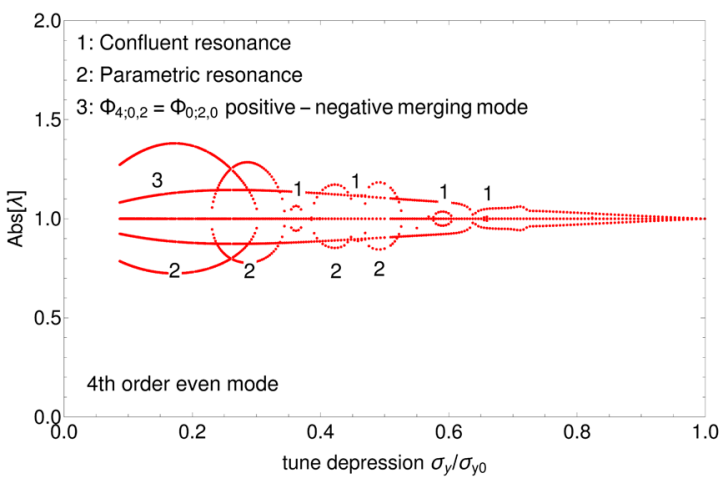

(a)

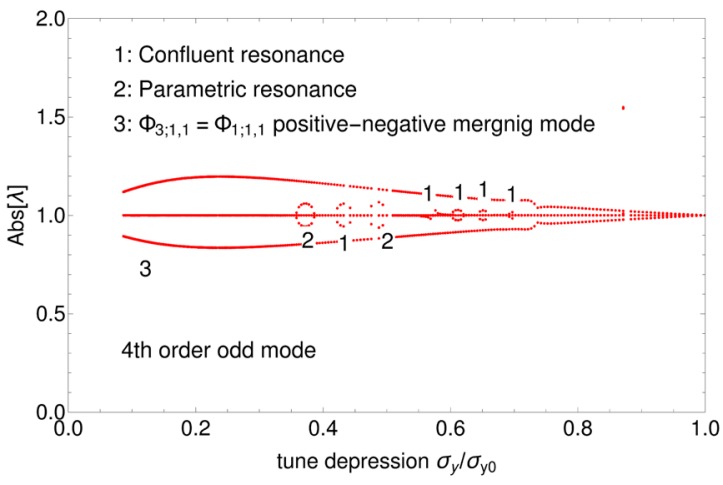

(c)

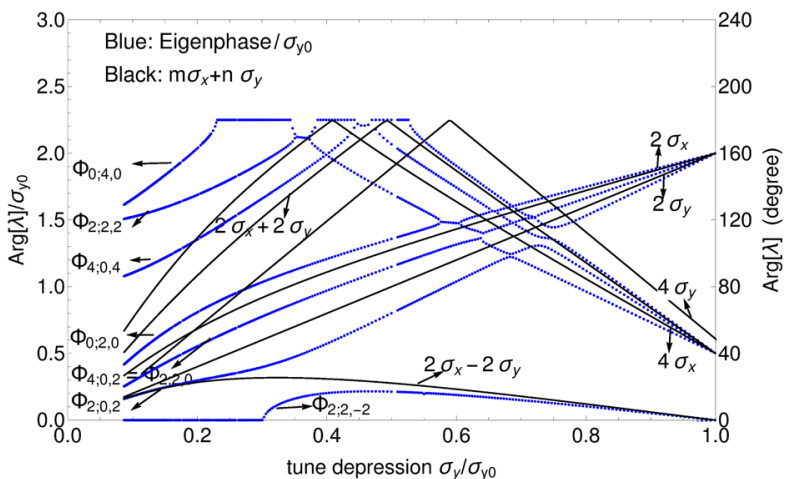

(b)

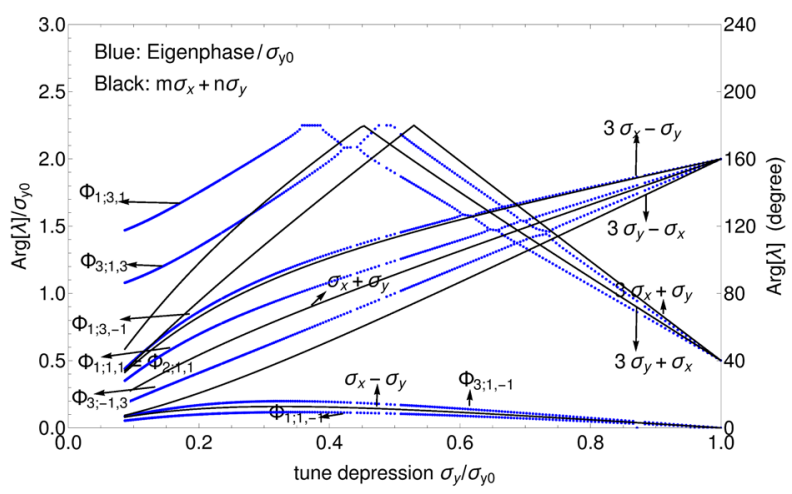

(d)

FIG. 4. Eigenvalues and eigenphases of the 4th order even, (a) and (b), and odd mode, (c) and (d), $v s$ tune depression $\sigma_{y} / \sigma_{y 0}$ for FODO channel (filling factor $\eta=0.5$, emittance ratio $\Gamma=1 / 2$ ) with the parameter $\sigma_{x 0}=\sigma_{y 0}=80^{\circ}$. 
TABLE IV. 4th order odd eigenmodes, eigenphases, and their driving terms.

\begin{tabular}{lcccc}
\hline \hline Periodic integral & Eigenphases & Driving term & Eigenphases (zero current) & Perturbed potential \\
\hline$I_{1 ; 1,1}$ & $\Phi_{1 ; 1,1}$ & $\cos \left[\left(\psi_{x}^{\prime}-\psi_{x}\right)+\left(\psi_{y}^{\prime}-\psi_{y}\right)\right]$ & $\sigma_{x 0}+\sigma_{y 0}$ & $A_{1} x^{3} y$ \\
$I_{1 ; 1,-1}$ & $\Phi_{1 ; 1,-1}$ & $\cos \left[\left(\psi_{x}^{\prime}-\psi_{x}\right)-\left(\psi_{y}^{\prime}-\psi_{y}\right)\right]$ & $\sigma_{x 0}-\sigma_{y 0}$ & $A_{1} x^{3} y$ \\
$I_{1 ; 3,1}$ & $\Phi_{1 ; 3,1}$ & $\cos \left[3\left(\psi_{x}^{\prime}-\psi_{x}\right)+\left(\psi_{y}^{\prime}-\psi_{y}\right)\right]$ & $3 \sigma_{x 0}+\sigma_{y 0}$ & $A_{1} x^{3} y$ \\
$I_{1 ; 3,-1}$ & $\Phi_{1 ; 3,-1}$ & $\cos \left[3\left(\psi_{x}^{\prime}-\psi_{x}\right)-\left(\psi_{y}^{\prime}-\psi_{y}\right)\right]$ & $3 \sigma_{x 0}-\sigma_{y 0}$ & $A_{1} x^{3} y$ \\
$I_{3 ; 1,1}$ & $\Phi_{3 ; 1,1}$ & $\cos \left[\left(\psi_{x}^{\prime}-\psi_{x}\right)+\left(\psi_{y}^{\prime}-\psi_{y}\right)\right]$ & $\sigma_{x 0}+\sigma_{y 0}$ & $A_{3} x y^{3}$ \\
$I_{3 ; 1,-1}$ & $\Phi_{3 ; 1,-1}$ & $\cos \left[\left(\psi_{x}^{\prime}-\psi_{x}\right)-\left(\psi_{y}^{\prime}-\psi_{y}\right)\right]$ & $\sigma_{x 0}+3 \sigma_{y 0}$ & $A_{3} x y^{3}$ \\
$I_{3 ; 1,3}$ & $\Phi_{3 ; 1,3}$ & $\cos \left[\left(\psi_{x}^{\prime}-\psi_{x}\right)+3\left(\psi_{y}^{\prime}-\psi_{y}\right)\right]$ & $-\sigma_{x 0}+3 \sigma_{y 0}$ & $A_{3} x y^{3}$ \\
$I_{3 ;-1,3}$ & $\Phi_{3 ;-1,3}$ & $\cos \left[-\left(\psi_{x}^{\prime}-\psi_{x}\right)+3\left(\psi_{y}^{\prime}-\psi_{y}\right)\right]$ & $\sigma_{x 0}+\sigma_{y 0}$ & $A_{1}^{(1)} x y$ \\
$I_{1 ; 1,1}^{(1)}$ & $\Phi_{1 ; 1,1}^{(1)}$ & $\cos \left[\left(\psi_{x}^{\prime}-\psi_{x}\right)+\left(\psi_{y}^{\prime}-\psi_{y}\right)\right]$ & $\sigma_{x 0}-\sigma_{y 0}$ & $A_{1}^{(1)} x y$ \\
$I_{1 ; 1,-1}^{(1)}$ & $\Phi_{1 ; 1,-1}^{(1)}$ & $\cos \left[\left(\psi_{x}^{\prime}-\psi_{x}\right)-\left(\psi_{y}^{\prime}-\psi_{y}\right)\right]$ & &
\end{tabular}

different external focusing characteristics, the parametric resonance stop band will shift and a new condition is required to avoid it.

Clearly, with higher order terms added in the potential polynomial $V$, more collective modes $I_{j ; k, l}$ could be constructed, leading to higher order stop bands. In fact, these stop bands would cover the whole parameter space if infinite orders of perturbation were plugged in. However, the effect of these higher order modes could be easily

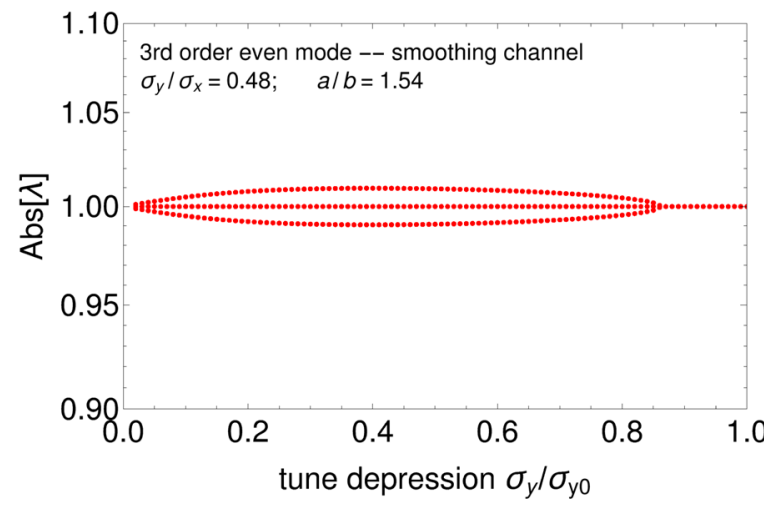

(a)

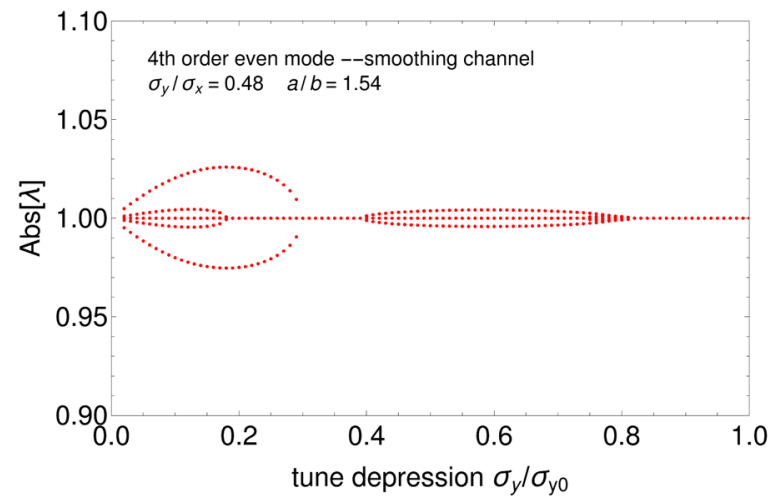

(c) washed out by the nonlinearity in the density profile, and when traversed, in general cause a small accumulative scattering effect.

\section{COLLECTIVE MODES WITH SMOOTH APPROXIMATION}

The smooth approximation is widely used in former studies in the collective mode analysis in accelerators.

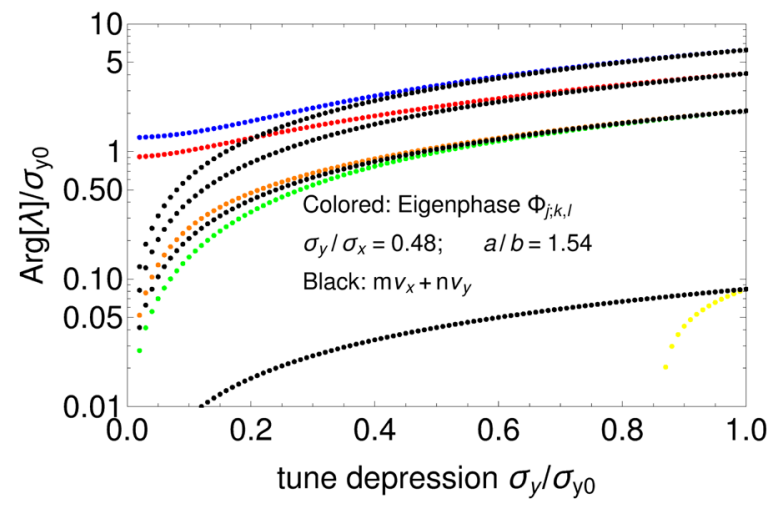

(b)

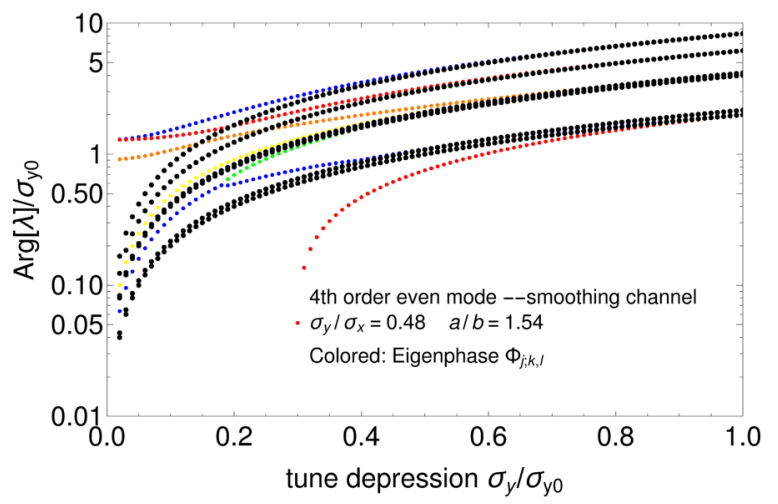

(d)

FIG. 5. The 3rd, (a) and (b), and the 4th, (c) and (d), order even modes eigenvalues and eigenphases as function of tune depression, when the external focusing is smoothed. The tune ratio is $\sigma_{y} / \sigma_{x}=0.48$, rms beam size ratio is $a / b=1.54$. It gives the same result as Fig. 5 and Fig. 7 shown in Ref. [6]. 


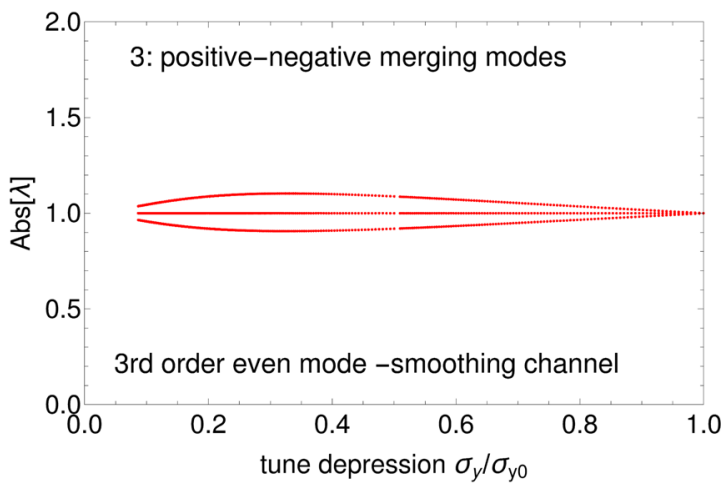

(a)

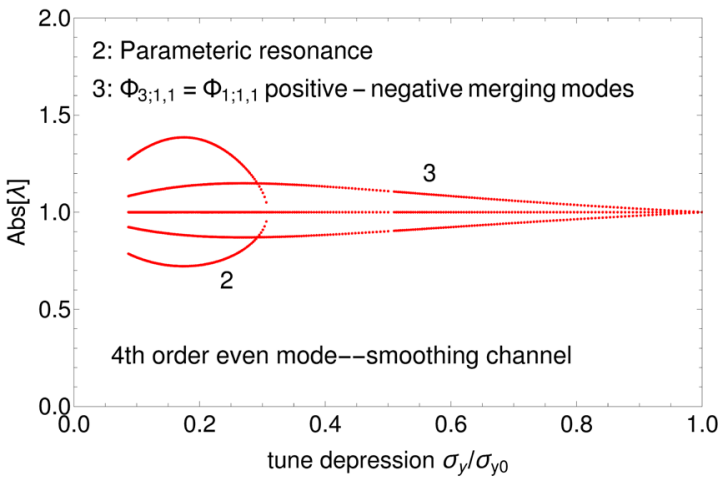

(c)

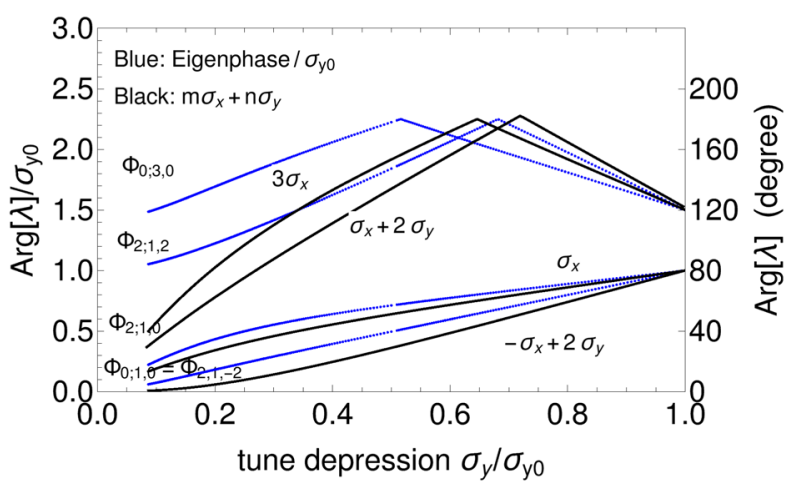

(b)

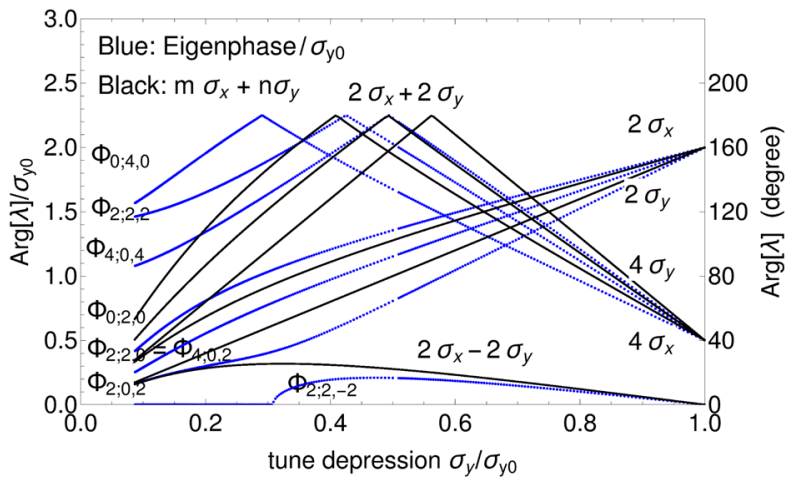

(d)

FIG. 6. Eigenvalues and eigenphase evolution of the 3 rd order even mode, (a) and (b), and the 4th order even mode, (c) and (d) $v s$ the tune depression in a smoothed constant focusing channel where fill factor $\eta=0.5$, emittance ratio $\Gamma=1 / 2$, phase advance $\sigma_{x 0}=\sigma_{y 0}=80^{\circ}$.

In the collective beam dynamics, the smooth approximation represents the constant focusing assumption [23], in which the matched beam rms size $R(s)$ is constant, which simplifies the derivation and analysis in most of the cases. With the self-consistent Vlasov-Poisson approach, Hofmann studied the dispersion relationship $[6,19]$ up to the fourth order systematically with smooth approximation. It is proved that the results from structure resonance discussed in this paper degenerates to Hofmann's result [6], if constant focusing channel is used. Fig. 5 shows two examples, obtained from structure resonance with constant focusing condition, and the eigenphases are plotted with logarithmic coordinate system. It shows the characteristics of the $3 \mathrm{rd}$ and 4 th order even modes versus tune depression. The colored curve represents the normalized eigenphase $\Phi_{j ; k, l} l \sigma_{y 0}$. The black curve represents the single particle resonance line $m \nu_{x} \pm n \nu_{y}\left(\nu=\sigma / \nu_{y 0}\right)$.

Two parametric stop bands are seen when the tune depression is below $0.82\left(\Phi_{3: 1,-2}=0^{\circ}\right)$ in Fig. 5(b) and the tune depression below $0.3\left(\Phi_{4 ; 2,-2}=0^{\circ}\right)$ in Fig. 5(d). The $\Phi_{j ; k, l}=0^{\circ}$ stop band is also considered as the modes merging with their conjugates. For the 4 th order even mode there are another two stop band components resulting from the (positive-negative) energy mode merging. These results obtained by structure resonance in a smoothed channel are the same as those given by Hofmann in Ref. [6].
Since the beam envelope is constant in the smoothed channel, the consequence is that beam only suffers from the parametric resonance $\Phi_{j ; k, l}=n * 180^{\circ}, n=0$ [24] and positive energy oscillation and negative energy oscillation modes merging resonance. As discussed, the latter is a peculiarity of the $\mathrm{KV}$ distribution and usually is ignored. Whereas, in the nonsmoothed channel, the parametric $\Phi_{j ; k, l}=n * 180^{\circ}, n \neq 0$ and confluent resonance between negative modes can also be excited, typical example is shown in Fig. 2.

Figure 6 shows the 3rd order and 4th order even modes obtained from a smooth approximation channel, which are with the same parameters as in Figs. 3 and 4. Comparing Fig. 3 and the two upper subfigures in Fig. 6, or comparing the two upper subfigures in Fig. 4 and the two lower subfigures in Fig. 6, show how the eigenvalues of the $3 \mathrm{rd}$ and 4 th even modes are affected by the smooth approximation. The parametric resonance stop band due to $\Phi_{2 ; 2,-2}=0^{\circ}$ of the 4th order modes agrees well in smoothed and nonsmoothed FODO channels. Extra stop bands appear in nonsmoothed FODO channel. The (positive-negative) merging mode $\Phi_{2 ; 1,-2}=\Phi_{0 ; 1,0}$ of the 3rd order and $\Phi_{4 ; 0,2}=\Phi_{0 ; 2,0}$ of the 4th order, in both smoothed and nonsmoothed FODO channels can be ignored as discussed. 


\section{DISCUSSION}

One question we need to answer from the former study [14] is that, if just considering the 2nd order even mode with symmetric beam condition $\left(\sigma_{x}=\sigma_{y}, \sigma_{x 0}=\sigma_{y 0}\right)$, why only parametric resonance takes place in the symmetric solenoid channels, and only confluent resonance takes place in the antisymmetric FODO channels. Now it is seen that the symmetry of the whole beam system plays as a key role. With an unsymmetric beam condition in the two degrees of freedom, both resonances can be excited, accompanied with stop band position shifting and width changing.

Again, the theory of structure resonance is based on linearized Vlasov-Poisson with $\mathrm{KV}$ initial distribution function. The eigenvalues discussed are always canonical conjugate pairs, which reflects the fact that the perturbed dynamics system is still in the frame of the Hamiltonian system. The 4D beam phase space can be distorted but the total area is still conserved. In numerical simulation, the $\mathrm{n}$-fold projection phase space pattern that leads to rms emittance growth or exchange between different degrees of freedom can be foreseen.

Another thing is that one may suspect that the $\delta$ function nature of $\mathrm{KV}$ distribution is responsible for these instabilities and the question arises as to how the unstable modes patterns change if the distribution is broadened and the loss cone in the $\mathrm{KV}$ beam is partially filled up. Certainly, nonlinear damping will be present to stabilize the beam, and "equilibrium" of a coasting beam would be reached as a compromise between resonance response, damping, and eventual saturation as the beam changes and moves out of the resonance. However, in a general sense, it is almost not possible to launch the study analytically, thus numerical simulations are always used to check the validity of theory prediction.

From the practical point of view, we propose that the accelerators should be designed or operated in the resonance free region, or with appropriate care when traversing resonances, to avoid emittance growth and beam halo leading to hardware damage. In future studies, we plan to show how beams are affected in the structure resonance stop bands with PIC simulations, and to investigate the effects of additional elements inside the transverse focusing period, such as rf gaps and additional types of focusing elements, and different forms of the transverse focusing lattice such as unequal focusing strengths.

\section{ACKNOWLEDGMENTS}

Great thanks to Mei Bai and King Y. Ng for helpful discussion. This work is supported by the Ministry of Science and Technology of China under Grant No. 2014CB845501.

[1] J. Stovall, J. Billen, S. Nath, H. Takeda, L. Young, D. Jeon, K. R. Crandall, and R. Shafer, in Proceedings of the 19th Particle Accelerator Conference, Chicago, IL, 2001 (IEEE, Piscataway, NJ, 2001), Vol. 1, p. 446.

[2] Y. Yamazaki, in Proceedings of the 2003 Particle Accelerator Conference, Portland, OR (IEEE, New York, 2003), p. 576.

[3] Z. Li, P. Cheng, H. Geng, Z. Guo, Y. He, C. Meng, H. Ouyang, S. Pei, B. Sun, J. Sun, J. Tang, F. Yan, Y. Yang, C. Zhang, and Z. Yang, Physics design of an accelerator for an accelerator-driven subcritical system, Phys. Rev. ST Accel. Beams 16, 080101 (2013).

[4] I. Hofmann, L. J. Laslett, L. Smith, and I. Haber, Stability of the Kapchinskij-Vladimirskij (K-V) distribution in long periodic transport systems, Part. Accel. 13, 145 (1983).

[5] C. L. et al., in 7th International Particle Accelerator Conference 2016, Busan, Korea, THOBAO2, 57th ICFA beam dynamics workshop HB2016, Maloe, Sweden 2016, THPM6Y01, DOI: 10.18429/JACoW-IPAC2016THOBA02.

[6] I. Hofmann, Stability of anisotropic beams with space charge, Phys. Rev. E 57, 4713 (1998).

[7] F. J. Sacherer, Ph.D. thesis, Lawrence Berkeley National Laboratory, 1968.

[8] F. Sacherer, A longitudinal stability criterion for bunched beams, IEEE Trans. Nucl. Sci. 20, 825 (1973).

[9] R. L. Gluckstern, Oscillation Modes in Two Dimensional Beams, Conf. Proc. C 700928, 811 (1970).

[10] R. L. Gluckstern, R. Chasman, and K. Crandall, Stability of Phase Space Distributions in Two Dimensional Beams, Conf. Proc. C 700928, 823 (1970).

[11] I. M. Kapchinskij and V. V. Vladimirskij, in Proceedings of the 9th International Conference on High Energy Accelerators (CERN, Geneva, 1959), p. 274.

[12] L. Laslett and L. Smith, Stability of intense transported beams, IEEE Trans. Nucl. Sci. 26, 3080 (1979).

[13] J. Struckmeier and M. Reiser, Theoretical studies of envelope oscillations and instabilities of mismatched intense charged-particle beams in periodic focusing channels, Part. Accel. 14, 227 (1983).

[14] C. Li and Q. Qin, Space charge induced beam instability in periodic focusing channel, Phys. Plasmas 22, 023108 (2015).

[15] M. Tabor, Chaos and Integrability in Nonlinear Dynamics (John Wiley \& Sons, New York, 1989).

[16] In rings, similar oscillation is considered as dipole mode.

[17] C. Li, Z. Liu, Y. Zhao, and Q. Qin, Nonlinear resonance and envelope instability of intense beam in axial symmetric periodic channel, Nucl. Instrum. Methods Phys. Res., Sect. A 813, 13 (2016).

[18] The collective modes with the eigenphase larger than the single particle resonance line (black curve), originating from the same value $m \sigma_{x} \pm n \sigma_{y}$, are termed as negative energy oscillation modes, and positive energy oscillation modes are termed as vice versa.

[19] I. Hofmann, G. Franchetti, O. Boine-Frankenheim, J. Qiang, and R. Ryne, Space charge resonances in two and three dimensional anisotropic beams, Phys. Rev. ST Accel. Beams 6, 024202 (2003).

[20] D. Jeon, L. Groening, and G. Franchetti, Fourth order resonance of a high intensity linear accelerator, Phys. Rev. ST Accel. Beams 12, 054204 (2009). 
[21] C. Li and Y. L. Zhao, Envelope instability and the fourth order resonance, Phys. Rev. ST Accel. Beams 17, 124202 (2014).

[22] In the former research [21], the comprehensive effect from collective 2 nd even mode and the 4 th order single-particlecore resonance are studied.
[23] Turn a time dependent system into time independent.

[24] Special case of confluent resonance, when $\Phi_{j ; k, l}=$ $\Phi_{j ; k, l}^{*}=0$, eigenphases and its canonical conjugate merges-(positive-negative) modes merging. 